ISSN: 2637-7764

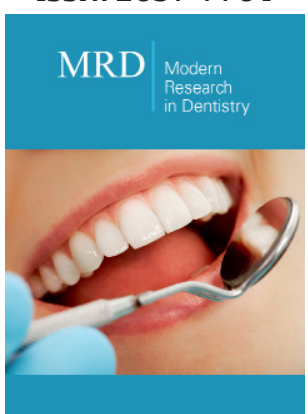

*Corresponding author: Fei Wang, Associate professor, Department of Orthodontics, Xi'an Jiao Tong University, China

Submission: 侮 June 11, 2021

Published: 侮 June 28, 2021

Volume 6 - Issue 4

How to cite this article: Meng-Yun Yang, Cheng $\mathrm{Hu}$, Janvier Habumugisha, XiaoJuan Gong, Fei Wang, et al. Correction of Maxillary Occlusal Plane Deviation with the Aid of Miniscrew Device. Mod Res Dent. 6(4). MRD. 000642. 2021.

DOI: 10.31031/MRD.2021.06.000642

Copyright@ Fei Wang, This article is distributed under the terms of the Creative Commons Attribution 4.0 International License, which permits unrestricted use and redistribution provided that the original author and source are credited.

\section{Correction of Maxillary Occlusal Plane Deviation with the Aid of Miniscrew Device}

Meng-Yun Yang, Cheng Hu, Janvier Habumugisha, Xiao-Juan Gong, Kun-Hong Bai, Bo Cheng, Meng-Qi Han, Shu-Yu Ma, Fei Wang*

Department of Orthodontics, College of Stomatology, Xi'an Jiao Tong University, P.R. China

\begin{abstract}
The therapy of the maxillary occlusal plane deviation differs depending on the etiology and pathogenesis. Canting caused by extruded teeth can be easily corrected with fixed orthodontic appliances combined with miniscrew anchorages. In this case, the canted maxillary occlusal plane was corrected by intruding extruded teeth with fixed orthodontic appliances combined with miniscrew anchorages, and a customized retainer was produced to prevent relapse.
\end{abstract}

Keywords: Occlusal plan deviation; Miniscrew anchorages; Retainer

\section{Introduction}

The maxillary occlusal plane deviation, also known as the canted maxillary occlusal plane, is one of the most frequent orthodontic disorders. Treatment varies to the point where surgical intervention may be required [1]. The Cant of the occlusal plane has been linked to a variety of etiologies. Canted occlusal planes caused by unilaterally extruded maxillary molars or uneven mandibular vertical development are common in patients with facial asymmetry [2]. According to Proffit et al. [3] "roll deformity" results from the orientation of the jaws and teeth rather than their position.

Since the introduction of miniscrew appliances into orthodontic practices, this equipment has played an essential role in resolving orthodontic difficulties that seem impossible to manage with conventional orthodontic equipment alone [4]. They can, for example, provide maximum anchorage, intrusion of elongated teeth, and other types of actions [5].

In this case report, we discuss a novel method for correcting a maxillary occlusal plane deviation with a miniscrew; a novel retention technique was also considered to assist in managing this case.

\section{Case Presentation}

A 31-year-old male patient complained of an occlusal plane deviation in the maxillary front teeth in January 2018. There was no history of orthodontic treatment, no family history of facial asymmetry, and no history of trauma; the problem developed approximately 17 years.

\section{Clinical examination}

1. Extraoral examination: mild asymmetry of frontal facial view (fullness at right side); normal of the profile view (Figure 1A).

2. Intraoral examination: permanent dentition; mild crowding in the upper and lower jaw; Class I relationship of molar and canine in both sides; upper and lower midlines were $1 \mathrm{~mm}$ shift to the right, and $1 \mathrm{~mm}$ shifts to the left respectively; $8 \mathrm{~mm}$ of an anterior overbite, $2 \mathrm{~mm}$ of anterior overjet.

3. TMJ check: no tenderness and clinking sound on both sides; regular opening and closing. 

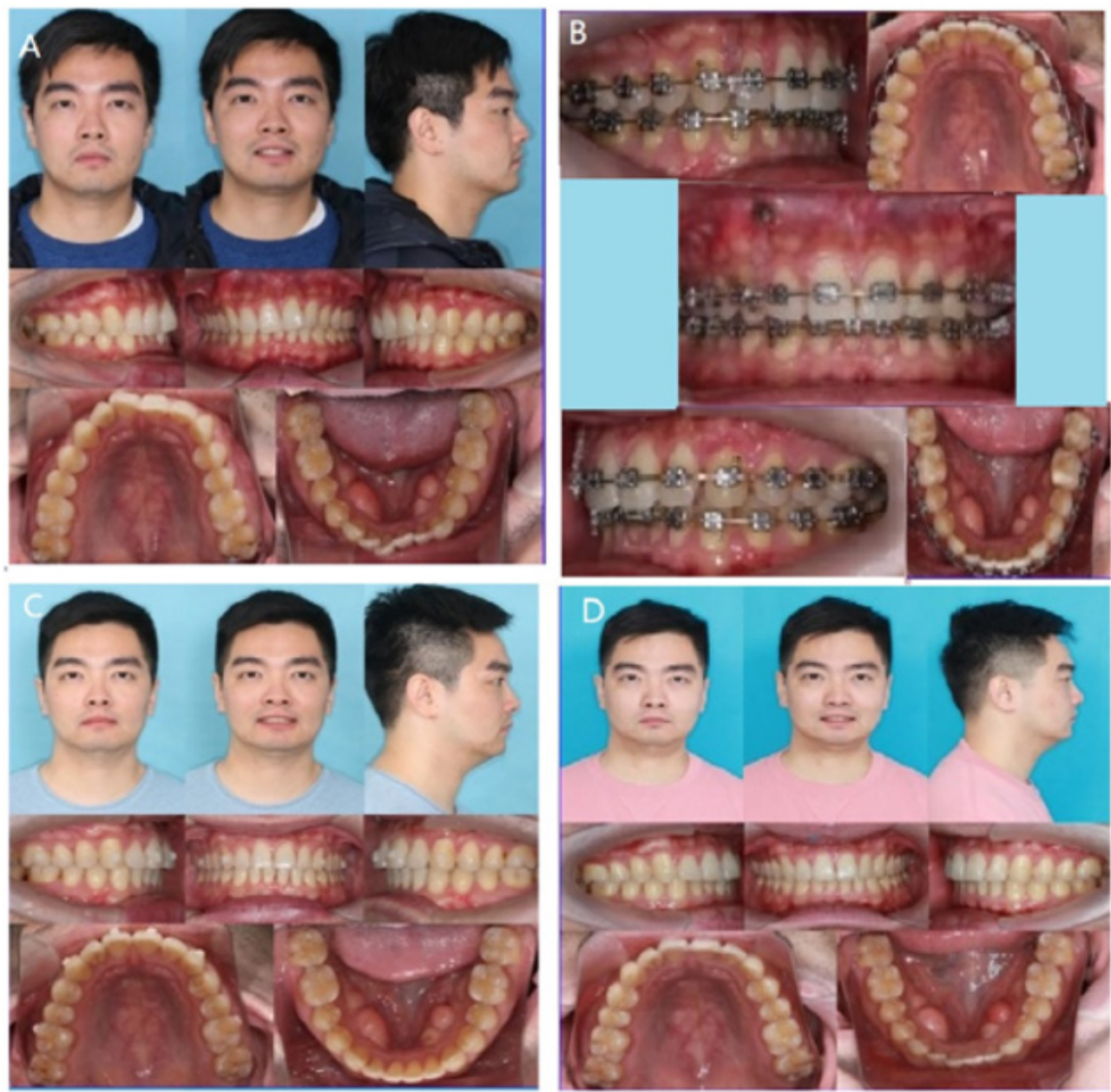

\section{Figure 1:}

A. Facial and intraoral view at the beginning of the correction,

B. Intraoral view after 9 months of treatment,

C. C: Intraoral view at the end of treatment

D. D: Facial and intraoral photographs after one year of retention.

\section{Radiography}

1.There are no congenitally missing teeth, supernumerary teeth, or four third molars. The height of the alveolar bone was located at the cementoenamel junction, the mandibular ramus and bodies were symmetrical (Figure 2A).
2. The analysis of cephalometric radiography before treatment was shown in Table 1.

3. CBCT shown asymmetry in bilateral condyle shape, size. The condylar cortex was continuous (Figure 2B).

Table 1: Cephalometric data before and after treatment and one year post retainer.

\begin{tabular}{|c|c|c|c|c|}
\hline Measurements & Normal Ranges & Before Treatment & After Treatment & One Year Post Retainer \\
\hline SNA & $82.8 \pm 4.0$ & 83.4 & 82.1 & 82.3 \\
\hline SNB & $80.1 \pm 3.9$ & 82.2 & 79.9 & 80.9 \\
\hline ANB & $2.7 \pm 2.0$ & 1.2 & 2.1 & 1.4 \\
\hline SND & $77.3 \pm 3.8$ & 80.6 & 78.3 & 79.5 \\
\hline Wits & $-1.1 \pm 2.4$ & -2.2 & 1.7 & -0.3 \\
\hline OP-SN & $16.1 \pm 5.0$ & 13 & 11.3 & 11.8 \\
\hline PO-NB (mm) & $1.0 \pm 1.5$ & 5.7 & 4.8 & 5.2 \\
\hline GOGN-SN & $32.5 \pm 5.2$ & 23.6 & 28.4 & 24.4 \\
\hline
\end{tabular}




\begin{tabular}{|c|c|c|c|c|}
\hline Mp-FH(FMA) & $31.3 \pm 5.0$ & 23.6 & 28.2 & 23.7 \\
\hline FMIA & $54.9 \pm 6.1$ & 67.4 & 28.6 & 62.6 \\
\hline U1-NA & $22.8 \pm 5.7$ & 19.6 & 5.7 & 5.8 \\
\hline U1-NA (mm) & $5.1 \pm 2.4$ & 3.2 & 23.2 & 22.9 \\
\hline L1-NB & $30.3 \pm 5.8$ & 18.6 & 3.9 & 3.6 \\
\hline L1-NB (mm) & $6.7 \pm 2.1$ & 1.5 & 91.2 & 93.6 \\
\hline IMPA & $93.9 \pm 6.2$ & 90 & & \\
\hline
\end{tabular}

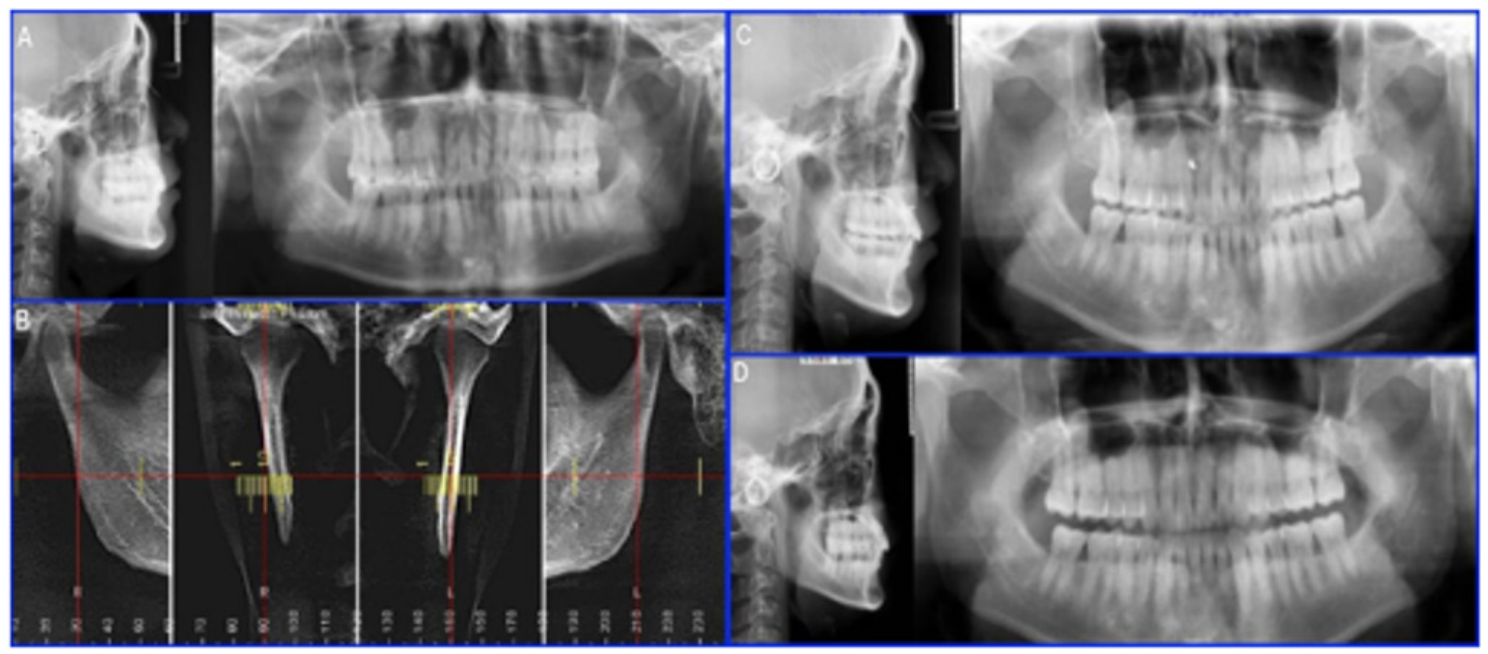

Figure 2:

A. Initial X-ray (lateral and panoramic),

B. Initial CBCT illustrating Temporal Mandibular Joint,

C. X-ray (lateral and panoramic) at the end of treatment,

D. X-ray (lateral and panoramic) after one year of the retention period.

\section{Model measurements}

Crowding: $2 \mathrm{~mm}$ on the maxillary arch, $2 \mathrm{~mm}$ on the mandibular arch; Bolton ratio for anterior teeth: $80.2 \%$, overall: $92.8 \%$; mandibular curve of Spee: $2 \mathrm{~mm}$ (Figure $3 \mathrm{~A}$ ).

\section{Diagnosis}

1. Soft tissue: frontal facial asymmetry, fullness at the right side; normal profile.

2. Skeletal tissue: Skeletal Class I in the sagittal direction and flat angle of mandibular plane in the vertical direction.

3. Dental: Angle Class I; crowded dentition; the maxillary occlusal plane was tilted (high in the left side and low in the right side); the deep anterior overbite; the midline deviation.

\section{Treatment plan}

The MBT appliance was utilized to align the upper and lower dentition in a non-extraction treatment. To correct the inclination of the maxillary occlusal plane, a miniscrew was implanted in the right maxillary.

\section{Process}

Bonding MBT brackets and buccal tubes to teeth 17-27, 37-47. The order of the orthodontic wires was as follows: Initial alignment wire was $0.012 "$, followed by $0.014 ", 0.016 "$, and 0.018 " nickeltitanium round wire, $0.016 \times 0.022$ " nickel-titanium rectangle wire, $0.017 \times 0.025^{\prime \prime}$ stainless steel wire, and $0.018 \times 0.025^{\prime \prime}$ stainless steel rectangle wire.

The crowding and lingual inclination of upper anterior teeth were corrected after the eighth months of treatment. Adequate alignment was accomplished; further, the upper midline was corrected, simultaneously, $1 \mathrm{~mm}$ space was established between 13 and 23 teeth. The crowding of the lower anterior teeth was not totally alleviated, however. To alleviate the crowding, all mandibular incisors underwent interproximal enamel reduction (IPR).

An $11 \mathrm{~mm}$ titanium alloy miniscrew (Ningbo Cibei Medical Instruments Co., Ltd., China) was planted between 12 and 13 at the $9^{\text {th }}$ month of treatment. The miniscrew was to intrude the extruded teeth and correct the canted maxillary occlusal plane. Additionally, a reverse curve of spee wire was used to the lower jaw to help correct a deep overbite in the mandibular anterior teeth (Figure 1B). 

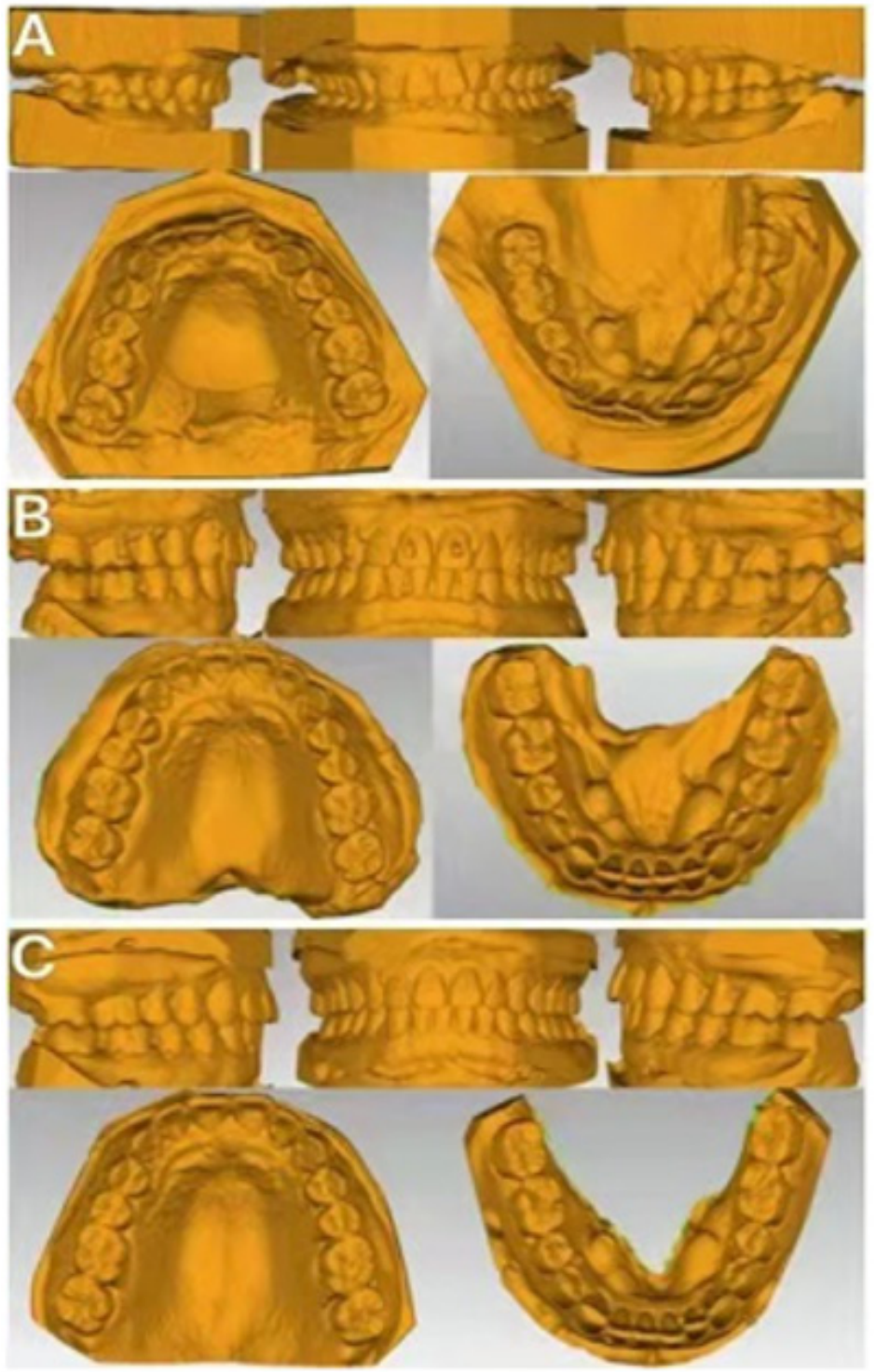

Figure 3:

A. Initial model,

B. Scanned model at the end of treatment,

C. scanned model after one year of the retention period.

The tilted maxillary occlusal plane was improved at the $12^{\text {th }}$ month, the $1 \mathrm{~mm}$ gap between 13 and 23 was closed, the deviation of the lower arch midline was corrected, and the maxillary miniscrew and arch wire were rigidly ligated. Intermaxillary traction was initiated for 1 month after 13 months of therapy to enhance overbite and ultimate occlusal relationship realignment. Patients wear retainers after 19 months. This case was treated from January 2018 until August 2019. Following the removal of orthodontic appliances, a customized orthodontic retainer with the adhesive resin attachments was created (Figure 1C \& Figure 3B). The patient was instructed to wear it for a year. Panoramic and cephalometric x-rays were obtained (Figure 2C). One year later, the patient returned to the clinic to follow up. The patient was given both a vacuum-formed retainer and a Hawley retainer at the same time. The patient was told to wear a vacuum formed retainer during the day and a Hawley retainer at night.

\section{Treatment result}

After the treatment, dentitional alignment was achieved. Furthermore, good occlusal relationships were achieved. The inclination of the occlusal plane was corrected, and the midline deviations in the upper and lower jaws were corrected. One year after treatment, the occlusal relationship was stable. There were slight signs of relapse in the maxillary occlusion plane (Figures 1D \& 3C). There was no significant change in cephalometry between pre and post-treatment and one year post retention (Figures 2D \& 4) (Table 1). 


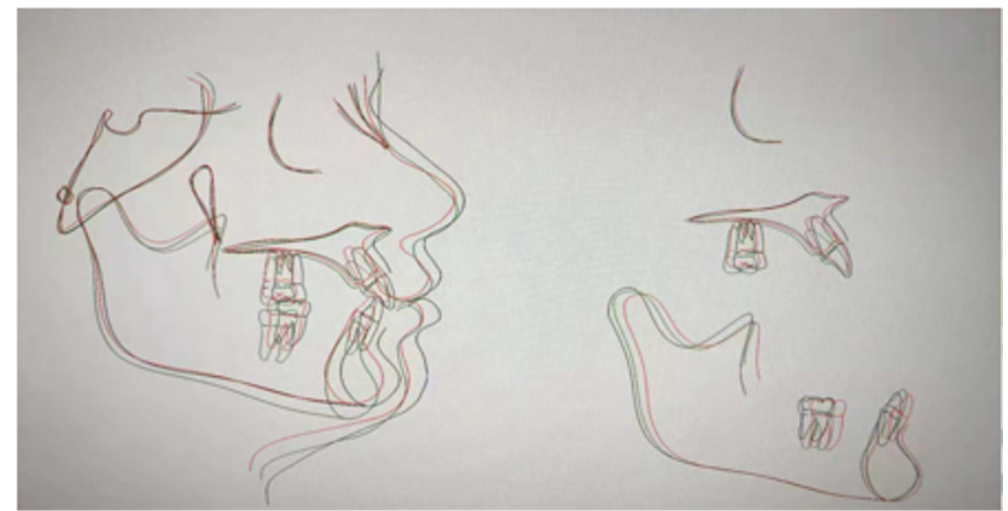

Figure 4: superimposition of initial, after treatment, and after one year of the retention period.

Black: initial Green: after treatment red: one-year post retention.

\section{Discussion}

Occlusal plane deviation that manifests as bilateral occlusal plane asymmetry is a common malocclusion. The following are the important causes of occlusal plane deviation: the unequal vertical development of the jaw on the left and right sides; the development of alveolar bone in the right and left posterior teeth is severely asymmetrical, leading to occlusal plane deviation, mandibular deviation, and facial asymmetry. The occlusal plane deviation caused by the asymmetry of the skeletal vertical growth in both sides necessitates a combination of orthodontic and orthognathic surgery to correct.

The patients with occlusal plane tilt can also be managed by multiple methods such as miniscrew anchorage [2], multi-loop on the rectangular archwire, auxiliary intrusion archwire, vertical traction methods, etc.

In this case, we used mini-screw anchoring to intrude the elongated teeth. The intrusive effectiveness attained was quite high, and the patients were comfortable during treatment; also, oral hygiene may be readily maintained. The use of miniscrew anchorage for intrusion in clinical orthodontic practice is a very good technique [6,5].

Periodontal tissue has its own specificity, instability, and relapse can occur at any time after orthodontic treatment. Therefore, it is important to avoid relapse during the retention period. There are several reasons resulting in relapse. The main ones include periodontal tissue rearrangement, muscular function maladjustment and lack of adequate retention $[1,7,8]$.

The patient must wear the proper retainer to decrease the teeth movement and recurrence rate. The retainers currently employed were Hawley retainers, vacuum-formed retainers, and fixed lingual retainers. Each of the retainers has advantages and disadvantages [9-11]. Previous research discovered that conventional retainers could give good long-term clinical stability in orthodontic cases, including extraction cases [12]. In this example, we primarily carried out the vertical intrusion for the anterior maxillary teeth. However, Traditional retainers show insufficient vertical control. We used the resin attachments of the invisible appliance as a guide and created a customized retainer. There was no recurrence of occlusal plane deviation one year after treatment. The customized film retainer was a successful method and deserved to be promoted in clinical applications.

\section{Conclusion}

The maxillary occlusal plane deviation caused by extruded teeth were easily corrected combined with the regular orthodontic equipment with the miniscrew anchorage; however, post-treatment retention should be carefully planned.

\section{Conflict of Interest}

The authors report no conflict of interest.

\section{References}

1. Vagdouti G, Karvouni E, Bitsanis E, Koletsi D (2019) Objective evaluation of compliance after orthodontic treatment using Hawley or vacuumformed retainers: A 2-center randomized controlled trial over a 3-month period. Am J Orthod Dentofacial Orthop 156(6): 717-726.e2.

2. Jeon YJ, Kim YH, Son WS, Hans MG (2006) Correction of a canted occlusal plane with miniscrews in a patient with facial asymmetry. Am J Orthod Dentofacial Orthop 130(2): 244-252.

3. William BE, Profit R, Henry W (2019) The etiology of orthodontic problems: Contemporary orthodontics. In: $\left(6^{\text {th }}\right.$ edn), Elsevier, St Louis, MO, Mosby, Missouri, USA, pp: 129-33.

4. Antoszewska Smith J, Sarul M, Łyczek J, Konopka T, Kawala B (2017) Effectiveness of orthodontic miniscrew implants in anchorage reinforcement during en-masse retraction: A systematic review and meta-analysis. Am J Orthod Dentofacial Orthop 151(3): 440-455.

5. Sherwood KH, Burch J, Thompson W (2003) Intrusion of supererupted molars with titanium miniplate anchorage. Angle Orthod 73(5): 597601. 
6. Park YC, Lee SY, Kim DH, Jee SH (2003) Intrusion of posterior teeth using mini-screw implants. Am J Orthod Dentofacial Orthop 123(6): 690-694.

7. MB Ackerman, MS McRae, WH Longley (2009) Microsensor technology to help monitor removable appliance wear. Am J Orthod Dentofacial Orthop 135(4): 549-551.

8. Thilander B (2000) Orthodontic relapse versus natural development Am J Orthod Dentofacial Orthop 117(5): 562-563.

9. Johnston CD, Littlewood SJ (2015) Retention in orthodontics. Br Dent J 218: $119-122$.
10. Gardner GD, Dunn WJ, Taloumis L (2003) Wear comparison of thermoplastic materials used for orthodontic retainers. Am J Orthod Dentofacial Orthop 124(3): 294-297.

11. Ramazanzadeh B, Ahrari F, Hosseini ZS (2018) The retention characteristics of Hawley and vacuum-formed retainers with different retention protocols. J Clin Exp Dent 10(3): e224-e231.

12. Edman Tynelius G, Petrén S, Bondemark L, Lilja Karlander E (2015) Fiveyear postretention outcomes of three retention methods-A randomized controlled trial. Eur J Orthod 37(4): 345-353. 